\title{
Ballistocardiography to identify high left atrial pressure in patients with heart failure
}

\author{
Li Zhang ${ }^{1,2}$, Peiwei Cai ${ }^{3}$, Yinlong Deng ${ }^{1}$, Jiumin Lin $^{4}$, Zhengpei Chu ${ }^{5}$, Qingfeng Shi ${ }^{5}$ Fei Ye $^{5}$, \\ Junhao $\mathrm{Hu}^{5}$, Chao Yang ${ }^{5}$, Pengyang $\mathrm{Li}^{6}$, Shaochun Zhuang ${ }^{7}$, Bin Wang ${ }^{1,2}$ \\ ${ }^{1}$ Department of Cardiology, the First Affiliated Hospital of Shantou University Medical College, Shantou, China; ${ }^{2}$ Clinical Research Center, the First \\ Affiliated Hospital of Shantou University Medical College, Shantou, China; ${ }^{3}$ Ultrasound Division, the First Affiliated Hospital of Shantou University \\ Medical College, Shantou, China; ${ }^{4}$ Department of Hepatology and Infectious Diseases, the Second Affiliated Hospital of Shantou University Medical \\ College, Shantou, China; ${ }^{5}$ DARMA Lab, Shenzhen, China; ${ }^{6}$ Department of Medicine, Saint Vincent Hospital, Worcester, MA, USA; ${ }^{7}$ Cardiostory \\ Inc., Lewes, DE, USA \\ Contributions: (I) Conception and design: B Wang, L Zhang; (II) Administrative support: B Wang, P Cai; (III) Provision of study materials or \\ patients: L Zhang, P Cai, Y Deng; (IV) Collection and assembly of data: Y Deng, L Zhang; (V) Data analysis and interpretation: Y Deng, J Lin; (VI) \\ Manuscript writing: All authors; (VII) Final approval of manuscript: All authors. \\ Correspondence to: Bin Wang, MD, PhD. Department of Cardiology, the First Affiliated Hospital of Shantou University Medical College, 57 \\ Changping Road, Shantou 515041, China; Clinical Research Center, the First Affiliated Hospital of Shantou University Medical College, 57 \\ Changping Road, Shantou 515041, China. Email: wangbin_pku@126.com; Shaochun Zhuang. Cardiostory Inc., 16192 Coastal Highway, Lewes, DE \\ 19958, USA. Email: shawn.z@cardiostory.com.
}

Background: Due to a high prevalence and morbidity rate, heart failure (HF) constitutes an immense economic burden on the global health care system. An increase in left atrial pressure (LAP) precedes the occurrence of any HF symptoms. In this study, we applied a novel non-invasive method of ballistocardiography (BCG) to extract early diastolic ventricular vibration waves [the BCG-B3 index, which corresponds to the third heart sound (S3) at the end of the rapid filling phase of diastole]. This study evaluated the predictive value of the BCG-B3 index for LAP in HF patients.

Methods: A total of $83 \mathrm{HF}$ patients and 20 patients with underlying diseases were prospectively enrolled, and their cross-sectional BCG and echocardiography (ECHO) data were collected. BCG obtains a signal through a high-precision fiber-optic sensor placed on the patient's back. LAP or pulmonary capillary wedge pressure (PCWP) was estimated by the ratio of mitral inflow peak early diastolic velocity to the early diastolic velocity of the mitral annulus (E/e') or the Nagueh equation ( $\left.\mathrm{LAP}=1.24 \times \mathrm{E} / \mathrm{e}^{\prime}+1.9\right)$. To evaluate the diagnostic efficacy of the BCG-B3 index, a receiver operating characteristic (ROC) curve was plotted, and the area under the ROC curve (AUC) was calculated. The best cutoff value for the BCG-B3 index was determined by the maximum Youden index.

Results: The correlation coefficient between the BCG-B3 index and E/e' ratio was $0.51(\mathrm{P}<0.01)$. Under an optimal cutoff value of 55.13, the BCG-B3 index showed a positive consistency value of 0.93 , a negative consistency value of 0.53 , and an overall consistency value of 0.82 for identification of significantly elevated LAP.

Conclusions: The BCG-B3 index derived by noninvasive BCG using a built-in fiber-optic sensor has important diagnostic value for identifying significantly elevated LAP in HF patients with high accuracy. BCG examination is not limited by place or the doctor's experience. Therefore, BCG can provide timely assessments for HF patients, enabling early diagnosis and treatment.

Keywords! Ballistocardiography (BCG); atrial pressure; heart failure (HF)

Submitted May 24, 2021. Accepted for publication Jul 16, 2021.

doi: 10.21037/apm-21-1840

View this article at: https://dx.doi.org/10.21037/apm-21-1840 


\section{Introduction}

Heart failure (HF) imposes a heavy health burden on society. Currently, at least 26 million individuals have HF worldwide, and the prevalence rate is $\geq 10 \%$ among the population older than 70 years (1). In China, the latest epidemiological study of HF published in 2019 showed that there were 13.7 million patients with $\mathrm{HF}$ in residents aged $\geq 35$ years, and the prevalence rate is as high as $1.3 \%$. The prevalence rate has increased by $44 \%$ in the past 15 years (2). The admission rate of HF patients is a strong independent predictor for the mortality of $\mathrm{HF}$ patients (3). Therefore, preventing rehospitalization and reducing the mortality rate of $\mathrm{HF}$ patients are very important (4).

Current out-of-hospital monitoring methods for $\mathrm{HF}$ patients include remote monitoring of patients' selfreported symptoms and signs, daily weight monitoring $(5,6)$, natriuretic peptide level monitoring $(7)$, noninvasive bioimpedance monitoring (8), implantable bioimpedance monitoring (9), and implantable hemodynamic sensors (10-12). Large randomized controlled trials have shown that only an implantable pressure sensor can directly assess left ventricular filling pressure (LVFP), and other methods cannot predict the prognosis of HF patients (13). However, pressure sensor implantation is an invasive operation, and its cost is relatively high, which limits clinical application (14). In addition to pressure sensor implantation to monitor filling pressure, clinicians have also tried to use the third heart sound (S3) to monitor LVFP in HF patients. Traditionally, $\mathrm{S} 3$ is assessed by experienced clinicians through cardiac auscultation with a stethoscope. Relevant studies have confirmed that the heart sound signals acquired by the accelerometer embedded in implantable cardiac devices are credible (15). In 2017, animal models confirmed that S3 measured by implantable devices was significantly correlated with left atrial pressure (LAP) and had high specificity in identifying elevated LAP (16).

At present, the main indicator of $\mathrm{HF}$ management is monitoring the LVFP. The LVFP can be indicated by pulmonary capillary wedge pressure (PCWP), LAP, or left ventricular end-diastolic pressure (LVEDP). PCWP measured by floating catheter and LVEDP measured by left ventricular catheter are commonly used as the gold standard for evaluating LVFP in clinical practice (17). The elevated LAP usually occurs before any symptoms of the hemodynamic cascade, which ultimately aggravates HF (18). Therefore, early assessment of LAP in patients with cardiac insufficiency may contribute to early prediction of $\mathrm{HF}$.
Ballistocardiography (BCG) records chest vibrations when the heart beats and ejects blood to peripheral blood vessels $(19,20)$. Electrocardiogram (ECG) records the electrical activity change during each cardiac cycle by the ECG machine (21). The optical fiber has the advantages of lighter weight, better ability of anti-electromagnetic interference and higher sensitivity (more than 3 magnitude higher than the piezoelectric sensor) (22). In this study, a novel fiber optic vibration sensor was used to record BCG signals generated by thoracic vibration in a nonimplanted way. This study is the first research using BCG technique to record BCG signals of diastolic hemodynamics of human heart through the thoracodorsal axis. Through acquisition and analysis of BCG signals of thoracic vibration in 103 adults (with different heart diseases), early diastolic ventricular vibration waves recorded by BCG (the BCG-B3 index, which corresponds to S3 at the end of the rapid filling phase of diastole) were extracted, which are physiologically related to S3. Previous studies have not shown whether the BCG-B3 index can effectively identify elevated LAP. The purpose of this study was to investigate whether the BCG-B3 index is related to LAP and whether it can effectively identify elevated LAP in HF patients. We present the following article in accordance with the STARD reporting checklist (available at https://dx.doi.org/10.21037/ apm-21-1840).

\section{Methods}

\section{Study population}

This research project was approved by the Ethics Review Committee of the First Affiliated Hospital of Shantou University Medical College (No. 2019071). All patients provided written informed consent before participating in this clinical single-center diagnostic trial. All procedures performed in this study involving human participants were in accordance with the Declaration of Helsinki (as revised in 2013).

From January 2019 to January 2020, 103 patients attending the echocardiography (ECHO) department of the First Affiliated Hospital of Shantou University Medical College were prospectively and continuously enrolled according to pre-established inclusion and exclusion criteria, and their cross-sectional BCG and ECHO data were collected. The flow chart of this study is shown in Figure 1.

The inclusion criteria were as follows: patients with 


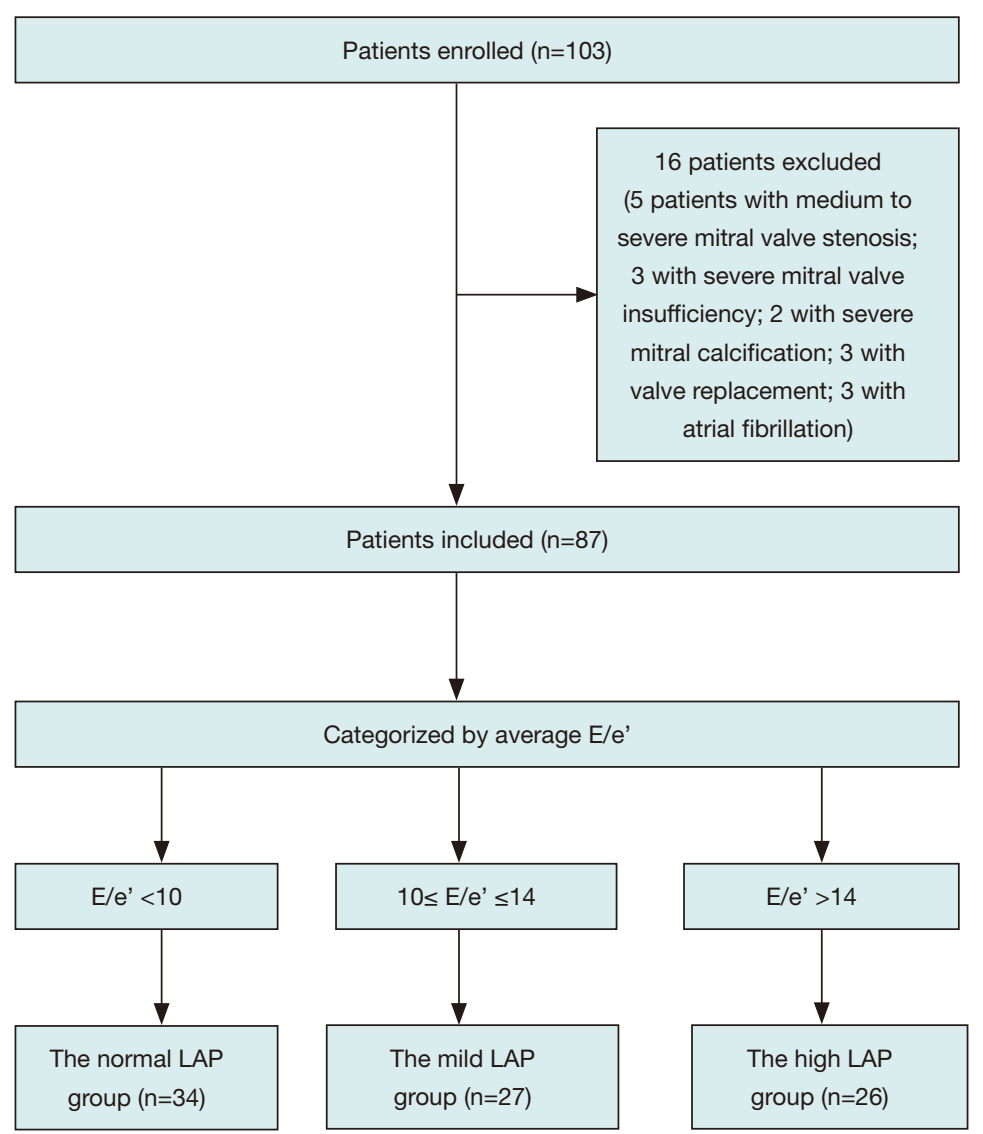

Figure 1 Study flowchart. E/e', mitral inflow peak early diastolic velocity to early diastolic velocity; LAP, left atrial pressure.

chronic HF and some patients with other underlying diseases. According to the 2016 European Society of Cardiology (ESC) guidelines for the diagnosis and treatment of acute and chronic HF (4), in addition to a relevant clinical history and signs, an HF diagnosis must also be confirmed by objective evidence, including at least one of the following: (I) an increased natriuretic peptide level; and (II) objective evidence of pulmonary or systemic congestion on imaging (e.g., high filling pressure by chest radiography or ECHO parameters) or by hemodynamic examination in the resting state or an active state [e.g., right heart catheterization (RHC) and pulmonary artery catheterization (PAC)].

The exclusion criteria were as follows: moderate or severe stenosis or insufficiency of a heart valve, patients with a prosthetic valve or mitral valvuloplasty; patients with severe mitral annulus calcification, patients with atrial fibrillation (AF) and congenital heart diseases. Changes in the size of and the pressure in the cardiac chambers of these patients were not caused by reduced diastolic function but by diseases in valves or structural changes in the heart. In AF patients, only the mitral inflow peak early diastolic velocity (E) can be obtained from transmitral blood flow, and relevant ultrasound parameters to evaluate diastolic function are lacking.

According to the study of Nagueh et al. and the 2016 ASE/EACVI guidelines $(17,23)$, LAP or PCWP can be calculated from the mean ratio of mitral inflow peak early diastolic velocity to the early diastolic velocity of the mitral annulus (E/e') or the Nagueh equation $\left(\mathrm{LAP}=1.24 \times \mathrm{E} / \mathrm{e}^{\prime}+1.9\right)$. Eighty-seven patients were divided into the normal LAP group $\left(\mathrm{E} / \mathrm{e}^{\prime}<10\right.$, $\mathrm{LAP}<14 \mathrm{mmHg})$, the mildly elevated LAP group $\left(10 \leq \mathrm{E} / \mathrm{e}^{\prime}\right.$ $\leq 14,14 \mathrm{mmHg} \leq \mathrm{LAP} \leq 18 \mathrm{mmHg}$ ), and the highly elevated LAP group (E/e' >14, LAP >18 mmHg).

\section{$B C G$}

Figure $2 A$ is a schematic diagram of the optical fiber. In Figure $2 B$, the fiber-optic sensor is embedded in a small 

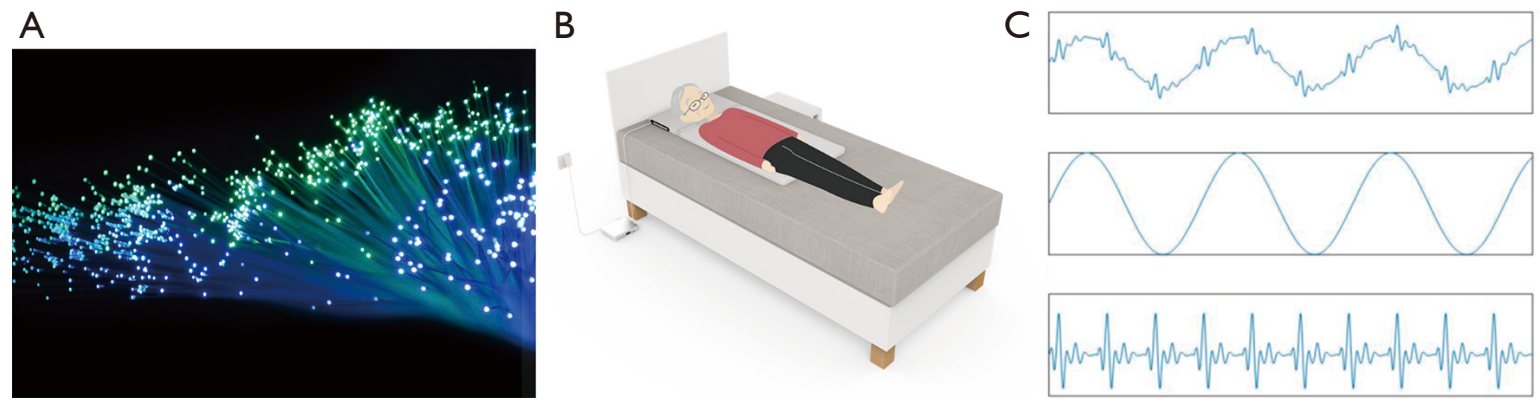

Figure 2 BCG equipment and signal based on optical fiber sensor. (A) Fiber-optic cables. (B) A patient lying on the device with the fiberoptic sensor and processor embedded. (C) Signal from the fiber-optic sensor. (C) Upper trace, a combination of respiratory movement and heartbeat signals; middle trace, isolation of the respiratory signal; and lower trace, signal of vibrations from heartbeats. This image is a simple depiction of the signal. Through amplification and other processes, more details can be observed from the signal. BCG, ballistocardiography.

mattress-like device under the patient's back. The patient is lying flat for 1 minute, and the BCG signals recorded at the thoracodorsal axis are transmitted wirelessly from the communication box to the diagnostic terminal. In Figure $2 C$ depicts the devices includes an electrocardiography (ECG) module, a phonocardiography (PCG) module, and a BCG module.

In the ECHO department, after communication with and obtaining informed consent from each patient, a 5 -minute BCG examination was performed with the builtin fiber-optic sensor after an ECHO examination. The BCG device was placed on a bed, and the patient laid supine on a mattress with the BCG assessment device. The ECG wires were connected to the left and right upper limbs and chest of the patient, PCG wires were attached to the patient's heart apex, and BCG sensors were attached to the patient's back. The BCG device simultaneously collected ECG, PCG, and BCG signals. The three signals are shown in Figure $3 A, B$.

By collecting and analyzing the BCG signals of 103 adults (with different heart diseases), we extracted the BCG-B3 index. In Figure 2, the fiber-optic vibration sensor (Figure $2 A$ ) is placed on the patient's back (Figure 2B). The fiber-optic vibration sensor obtains a composite signal of the patient's chest vibration from the back, which contains the chest vibration caused by the patient's respiration and the patient's BCG signal [Figure 2C (upper)]. The composite superimposed signal is preliminarily filtered to obtain the vibration signal of standard respiration [Figure $2 C$ (middle)] and the BCG signal [Figure 2C (lower)]. The BCG signal is further filtered, and the R peak of the QRS of the ECG signal is used as a reference point to divide the signal of multiple consecutive cardiac beats into multiple segments, and each segment contains only one cardiac beat. The BCG signal in each segment is normalized, i.e., the maximum amplitude of the systolic phase (between R and S2) in the segment is set to $100 \%$, and the amplitudes of the signal at other positions are correspondingly normalized (between 0 and $100 \%$ ), thus yielding multiple normalized amplitudes of the heartbeat signal in segments. After normalization, multiple heartbeat signals are superimposed to obtain the average signal of multiple heartbeats. Next, the R peak in the QRS signal in the ECG signal of each heartbeat is used as a reference time point, and the BCG signals of multiple heartbeats are superimposed to obtain average ECG, PCG, and BCG curves, as shown in Figure $3 A, B$. From the average BCG curve, the typical QRS signal can be identified, and the $\mathrm{S} 1$ and $\mathrm{S} 2$ signals can be identified from the PCG curve. The characteristics of three main signals can facilitate the division of cardiac cycle intervals. According to a physiological description, the period after S2 and before the ECG P wave belongs to early diastole. The maximum amplitude in early diastole is extracted and marked on the BCG curve, which is defined as the BCG-B3 index. After normalization, the amplitude of BCG-B3 is between 0 and $100 \%$. BCG-B 3 is the ratio of the amplitude of the position of $\mathrm{B} 3$ to the maximum amplitude of the systolic phase, and is a dimensionless relative value. The BCG-B3 index was automatically output by the BCG device, and the data were recorded and analyzed by a technician who did not have access to the clinical data and other hemodynamic data of the patients.

During early diastole, the heart completes early filling followed by a short rest period. The length of the rest 
A

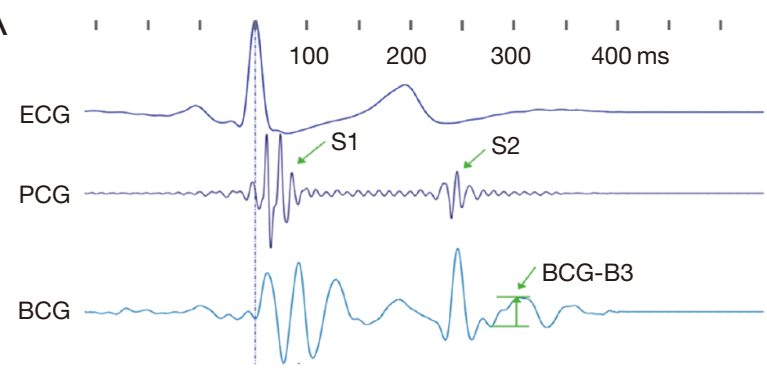

C

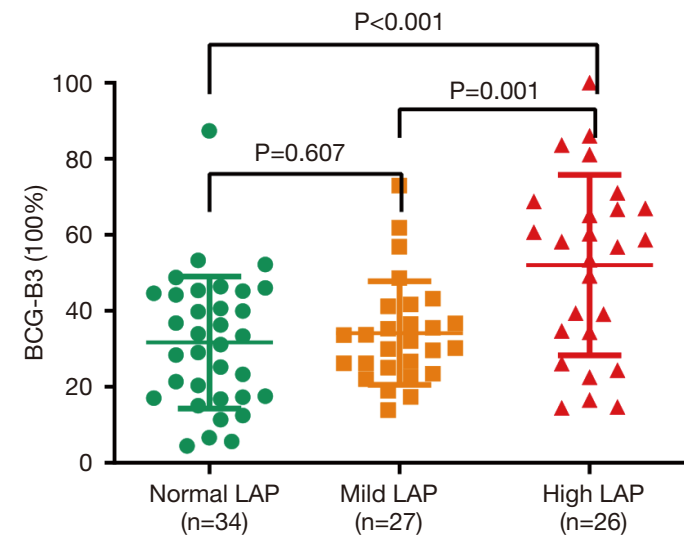

$E$

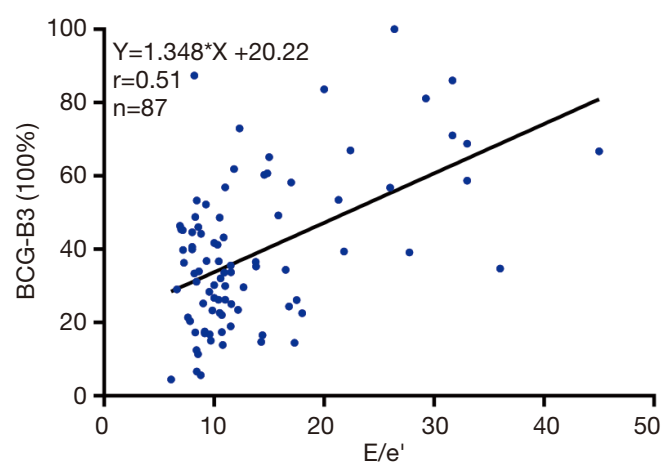

B

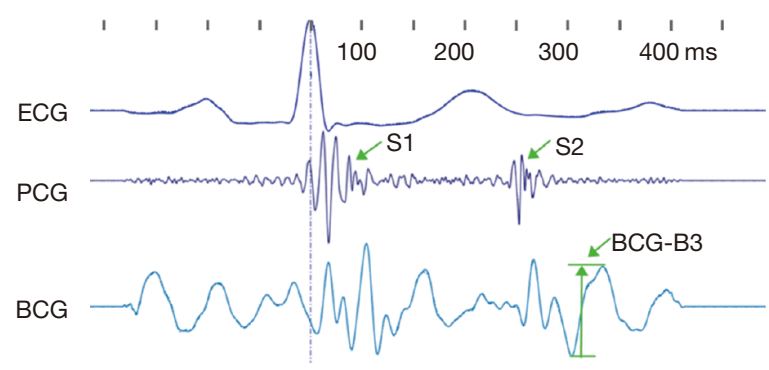

D

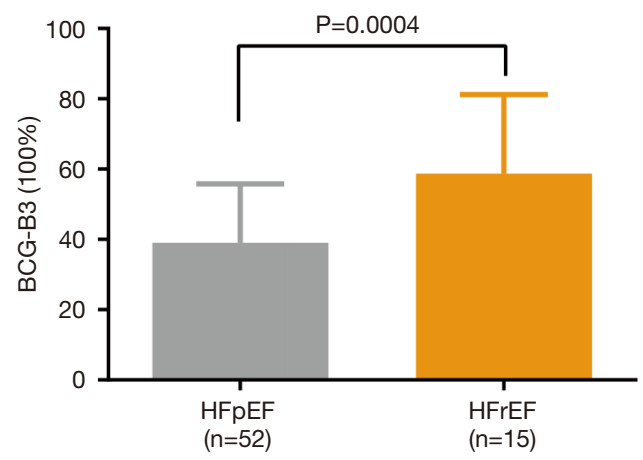

$\mathrm{F}$

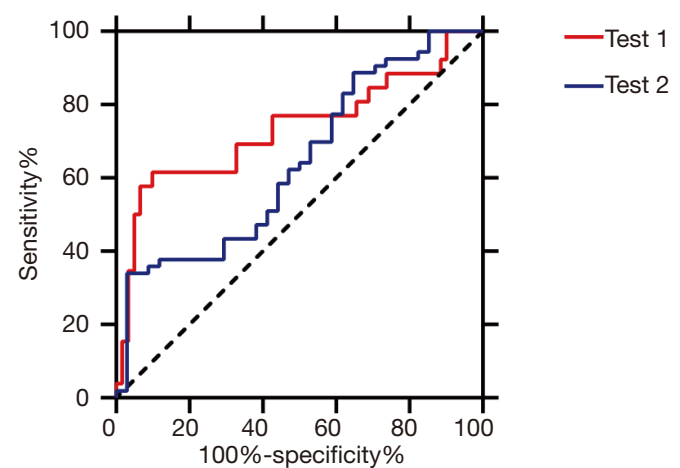

Figure 3 The BCG-B3 index identify high LAP. (A) Signal from a healthy subject with a lower BCG-B3 amplitude on the BCG signal. (B) Signal with higher BCG-B3 on BCG from a patient with high LAP. (C) Comparison of the BCG-B3 index among the three study groups. (D) Comparison of the BCG-B3 index between the HFrEF group and HFpEF group. (E) Relationship of the BCG-B3 index with the E/e' ratio. (F) ROC curve for the detection of high LAP (test 1) and mild LAP (test 2) using the BCG-B3 index. LAP was estimated by ECHO. BCG-B3, early diastolic ventricular vibration waves recorded by ballistocardiography; LAP, left atrial pressure; BCG, ballistocardiography; HFrEF, heart failure with a reduced ejection fraction; HFpEF, heart failure with a preserved ejection fraction; E/e' ratio, mitral inflow peak early diastolic velocity to the early diastolic velocity of the mitral annulus; ROC, receiver operating characteristic; ECHO, echocardiography; ECG, electrocardiogram; PCG, phonocardiography.

period is related to the total duration of heartbeats. The $\mathrm{E}$ of transmitral blood flow and the early diastolic velocity of the mitral annulus (e') in early diastole were recorded by ECHO.

\section{ECHO}

In the ECHO department, ultrasound images were collected using a Philips Sonos 5500 Ultrasound System and Doppler tissue imaging (DTI) software. ECHO image 
acquisition was performed in an offline analysis platform (MedEx) equipped with two-dimensional (2D) and Doppler by a technician who did not know the clinical data and hemodynamic data of the patient. The specific measurement methods are as follows.

Measurement method for the left ventricular ejection fraction (LVEF): the left ventricular endocardial margin was delineated on the four-chamber and two-chamber views of the heart, and the end-diastolic volume (EDV) and endsystolic volume (ESV) were calculated by the modified Simpson method (biplane method of disks). The LVEF was calculated using the EDV and ESV, and the formula is $\mathrm{LVEF}=(\mathrm{EDV}-\mathrm{ESV}) / \mathrm{EDV}$. Measurement method for mitral valve flow: in the apical four-chamber view, pulse Doppler was used to measure the forward flow velocity at the mitral valve orifice to assess left ventricular filling. During diastole, a sampling volume of 1 to $3 \mathrm{~mm}$ was placed on the cusps of the mitral valve to record a clear blood flow velocity profile, and the main measurement indicators included $\mathrm{E}$, mitral inflow peak late diastolic velocity (A), and the E/A ratio. DTI of the mitral annulus: pulsed DTI was used to measure mitral annulus velocity at the apical section. The sampling volume was placed at or within 1 $\mathrm{cm}$ of the attachment position of the ventricular septum in the mitral valve and the lateral wall. The measurement indicators included e' and the late diastolic velocity of the mitral annulus (a'). Measurement method for the left atrial volume index: the $2 \mathrm{D}$ method was used to delineate the blood-tissue interface on the apical four-chamber view or two-chamber view, and the left atrial volume was calculated using the biplane method of disks. Measurement method for peak tricuspid regurgitation velocity (VTR): continuous Doppler was used to measure the peak VTR during systole. In this study, the parameter e' is the average e' and E/e' is the average E/e'.

\section{Statistical analysis}

This study was a preliminary study of LAP identification by a BCG device; thus, a specific and accurate sample size cannot be calculated. Statistical analysis was performed using IBM SPSS 22.0. GraphPad Prism 8.3 was used to draw statistical graphs. Continuous variables are expressed as the mean \pm standard deviation (SD); categorical variables are expressed as $\mathrm{n}(\%)$. The Kolmogorov-Smirnov (K-S) test $(\mathrm{n}>50)$ and the Shapiro-Wilk $(\mathrm{S}-\mathrm{W})$ test $(\mathrm{n}<50)$ were used to test the normality of the continuous variables in each group. Before comparison, all variables in each group were tested for normality and homogeneity of variance. When comparing variables between two groups, if groups satisfied the tests of normality $(\alpha=0.05)$ and homogeneity of variance, the $t$-test was used; if one group showed a skewed distribution, the Mann-Whitney $\mathrm{U}$ test was used. When comparing variables among three groups, if groups met the tests of normality $(\alpha=0.05)$ and homogeneity of variance, one-way analysis of variance (ANOVA) was used. The least significant difference (LSD), Student-Newman-Keuls (SNK) test, and Dunnett test for post-hoc analysis were used to compare differences between groups. The KruskalWallis $\mathrm{H}$ test was used to compare differences among the three groups if any one variable did not pass the normality test. Pearson correlation analysis was used for the BCG-B3 index and the E/e' ratio since both variables showed a normal distribution. To investigate the diagnostic efficacy of the BCG-B3 index, a receiver operating characteristic (ROC) curve was used, and the area under the ROC curve (AUC) was calculated. The cutoff value of the BCG-B3 index was determined by the maximum Youden index. The consistency between the BCG-B3 index and ECHO parameters in identifying elevated LAP was measured using a matched fourfold table. $\mathrm{P}<0.05$ was considered statistically significant.

\section{Results}

\section{Patient population and clinical characteristics}

Among 103 patients, 16 were excluded (five with moderate and severe mitral stenosis, three with severe mitral valve insufficiency, two with severe mitral annulus calcification, three with valve replacement, and three with $\mathrm{AF}$ ) since BCG and ECHO cannot evaluate them correctly. Finally, 87 patients were fully assessed with BCG and ECHO. Thirty-four patients were included in the normal LAP group, including 14 patients with the New York Heart Association (NYHA) functional class I/II HF. None of these patients showed signs or symptoms of HF. The mildly elevated LAP group included 27 patients with NYHA functional class I-IV HF. These patients did not have pulmonary congestion-related symptoms. The significantly elevated LAP group consisted of 26 patients with NYHA functional class III-IV HF and pulmonary congestion-related symptoms or signs of varying degrees. All hypertension patients received $\beta$-blockers, calcium channel blockers, or both; patients with coronary heart disease (CHD) were treated with nitrate esters; and patients with diabetes were treated with hypoglycemic therapy. 
Table 1 Clinical characteristics in the study groups

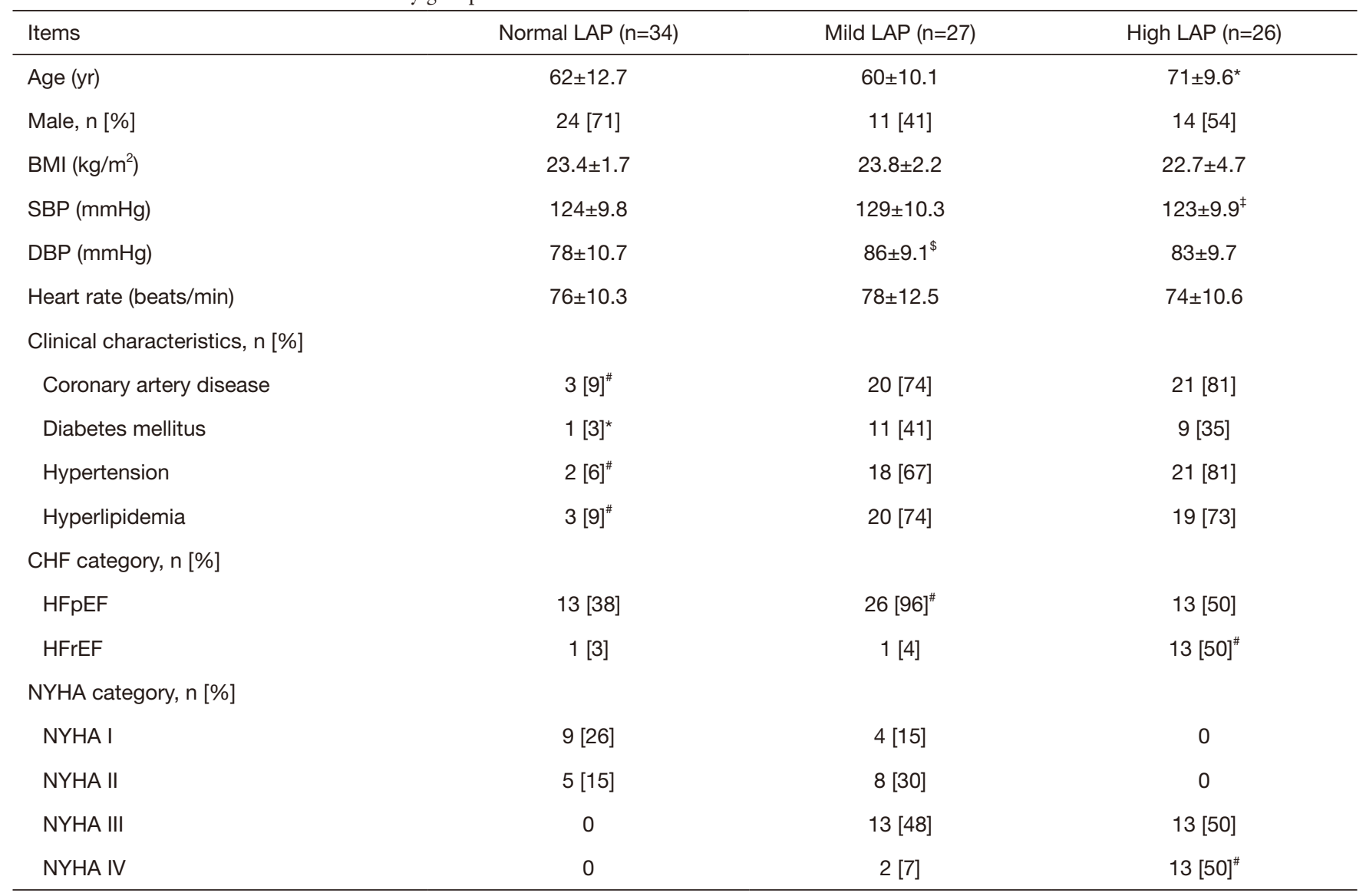

The mean \pm SD or the number of patients. ", $\mathrm{P}<0.001$ compared with the other two groups; * $\mathrm{P}<0.01$ compared with the other two groups;

$\$, \mathrm{P}<0.01$ compared with the normal LAP group; ${ }^{\ddagger}, \mathrm{P}<0.05$ compared with the mild LAP group. LAP, left atrial pressure; BMI, body mass index; SBP, systolic blood pressure; DBP, diastolic blood pressure; CHF, congestive heart failure; HFpEF, heart failure with a preserved ejection fraction; HFrEF, heart failure with a reduced ejection fraction; NYHA, New York Heart Association; SD, standard deviation.

The demographic and clinical characteristics of the three groups are shown in Table 1. No significant differences in sex composition, heart rate, and body mass index (BMI) were noted among the three groups (Table 1). Both the mildly and significantly elevated LAP groups had a high prevalence of clinically relevant diseases $(\mathrm{P}<0.01)$. Diastolic blood pressure (DBP) in the mildly elevated LAP group was higher than that in the other two groups $(\mathrm{P}<0.01)$. In the significantly elevated LAP group, the patients were older $(\mathrm{P}<0.01)$, and the ratios of heart failure with a reduced ejection fraction (HFrEF) patients and NYHA IV patients were higher than those in the other two groups $(\mathrm{P}<0.01)$.

\section{The BCG-B3 index and ECHO variables}

The distribution characteristics of the BCG-B3 and ECHO parameters in the three groups are shown in Table 2. The distribution of the BCG-B3 index among the three groups is shown in Figure 3C. No statistically significant difference in the BCG-B3 index was found between the normal and mildly elevated LAP groups $(\mathrm{P}=0.607)$. The difference in the BCG-B3 index between the normal and significantly elevated LAP groups was statistically significant $(\mathrm{P}<0.001)$. The difference in the BCG-B3 index between the mildly and significantly elevated LAP groups was statistically significant $(\mathrm{P}=0.001)$. Compared with the significantly elevated LAP group, the other two elevated LAP groups had significantly higher BCG-B3 indexes, E values, E/ A ratios, and $\mathrm{E} / \mathrm{e}$ ' ratios $(\mathrm{P}<0.01)$, and the $\mathrm{LVEF}$ and a' were significantly lower $(\mathrm{P} \leq 0.001)$. The e' followed the order of the normal LAP group $>$ the mildly LAP group $>$ significantly elevated LAP group, and the differences 
Table 2 BCG-B3 and echocardiographic variables in the study groups

\begin{tabular}{|c|c|c|c|}
\hline Items & Normal LAP $(n=34)$ & Mild LAP ( $\mathrm{n=27})$ & High LAP $(n=26)$ \\
\hline LVEF (\%) & $65 \pm 5.4$ & $67 \pm 7.3$ & $52 \pm 16^{\#}$ \\
\hline $\mathrm{E}(\mathrm{cm} / \mathrm{s})$ & $66 \pm 14.9$ & $71 \pm 12.9$ & $103 \pm 32.1^{\#}$ \\
\hline $\mathrm{A}(\mathrm{cm} / \mathrm{s})$ & $87 \pm 17.6$ & $93 \pm 16.0$ & $96 \pm 28.3$ \\
\hline$e^{\prime}(\mathrm{cm} / \mathrm{s})$ & $8.1 \pm 2.1^{\#}$ & $6.3 \pm 1.2^{\#}$ & $4.7 \pm 1.3^{\#}$ \\
\hline $\mathrm{a}^{\prime}(\mathrm{cm} / \mathrm{s})$ & $11.2 \pm 2.2$ & $10.9 \pm 1.9$ & $8.2 \pm 2.6^{\#}$ \\
\hline e'/a' ratio & $0.7 \pm 0.2$ & $0.6 \pm 0.1$ & $0.6 \pm 0.2$ \\
\hline E/e' ratio & $8.3 \pm 0.9^{\ddagger}$ & $11.1 \pm 1.0$ & $22.8 \pm 8.1^{\#}$ \\
\hline
\end{tabular}

The data presented are the mean \pm SD. ", $\mathrm{P} \leq 0.001$ compared with the other two groups; ${ }^{\prime}, \mathrm{P}<0.01$ compared with the other two groups;

${ }^{\ddagger}, \mathrm{P}<0.05$ compared with the mild LAP group. BCG-B3, early diastolic ventricular vibration waves recorded by ballistocardiography; LAP, left atrial pressure; LVEF, left ventricular ejection fraction; E, mitral inflow peak early diastolic velocity; A, mitral inflow peak Ite diastolic velocity; E/A ratio, early to late transmitral flow velocity; e', early diastolic velocity of the mitral annulus; a', late diastolic velocity of the mitral annulus; e'/a' ratio, mitral annulus early diastolic velocity to late diastolic velocity; E/e' ratio, mitral inflow peak early diastolic velocity of the mitral annulus.

Table 3 Relationships of the BCG-B3 index with 2D, Doppler echocardiographic, and DTI variables

\begin{tabular}{lcc}
\hline Items & $r$ & P value \\
\hline LVEF $(n=87)$ & 0.39 & $<0.01$ \\
Mitral E velocity $(n=87)$ & 0.46 & $<0.01$ \\
Mitral A velocity $(n=87)$ & -0.19 & $<0.01$ \\
Mitral E/A ratio $(n=87)$ & 0.50 & $<0.01$ \\
E/e' ratio $(n=87)$ & 0.51 & $<0.01$ \\
e' $(n=87)$ & -0.25 & 0.01 \\
a' $(n=87)$ & -0.43 & $<0.01$ \\
e'/a' ratio $(n=87)$ & 0.15 & 0.16 \\
VTR $(n=51)$ & 0.31 & $<0.01$ \\
LAMVI $(n=37)$ & 0.10 & 0.53 \\
PAsP $(n=51)$ & 0.26 & 0.06 \\
\hline
\end{tabular}

BCG-B3, early diastolic ventricular vibration waves recorded by ballistocardiography; 2D, two-dimensional; DTI, Doppler tissue imaging; LVEF, left ventricular ejection fraction; $E$, mitral inflow peak early diastolic velocity; $A$, mitral inflow peak late diastolic velocity; E/A ratio, early to late transmitral flow velocity; E/ e' ratio, mitral inflow peak early diastolic velocity to the early diastolic velocity of the mitral annulus; e', early diastolic velocity of the mitral annulus; a', late diastolic velocity of the mitral annulus; e'/a' ratio, mitral annulus early diastolic velocity to late diastolic velocity; VTR, tricuspid regurgitation velocity; LAMVI, left atrial maximal volume index; PAsP, pulmonary artery systolic pressure. were statistically significant $(\mathrm{P} \leqslant 0.001)$. No significant differences in the e'/a' ratio were found among the three groups. The distribution characteristics of the BCG-B3 index (Figure 3D) between the HFrEF group $(58.72 \pm 5.80)$ and heart failure with a preserved ejection fraction (HFpEF) group (39.00 \pm 2.32$)$ showed that the BCG-B3 amplitude in the HFrEF group was significantly higher than that in the HFpEF group, and a significant difference was identified between the two groups $(\mathrm{P}=0.0004)$.

\section{Relationships of the BCG-B3 index with ECHO variables}

The correlations between the BCG-B 3 index and the ECHO parameters are shown in Table 3, and the distribution of $\mathrm{r}$ is wide. The BCG-B3 index $[38.54 \pm 20.40$ (range, 4.44 to 100.00)] had the best correlation with the E/e' ratio, with an $\mathrm{r}$ value of $0.51(\mathrm{P}<0.01)$ (Figure $3 E$ ), followed by the E/A ratio, with an $\mathrm{r}$ value of $0.50(\mathrm{P}<0.01)$, and the largest negative correlation was found for a', with an $r$ value of $-0.43(\mathrm{P}<0.01)$. No correlations were found between the BCG-B3 index and the e'/a' ratio, pulmonary artery systolic pressure (PAsP), and LAMVI.

\section{Diagnostic test characteristics of the BCG-B3 index}

As shown in Table 4 and Figure 3F, when the BCG-B3 index identified significantly elevated LAP, under a best cutoff 
Table 4 Accuracy of diagnosing high LAP: total population

\begin{tabular}{lcr}
\hline Items & $\begin{array}{c}\text { Test 1: BCG-B3 identification } \\
\text { (high vs. normal LAP) }\end{array}$ & $\begin{array}{c}\text { Test 2: BCG-B3 identification } \\
\text { (mild + high vs. normal LAP) }\end{array}$ \\
\hline Best cutoff & 55.13 & 48.96 \\
Positive consistency rate & $0.93(0.84-0.98)$ & $0.29(0.19-0.41)$ \\
Negative consistency rate & $0.53(0.33-0.73)$ & $1.00(0.73-1.00)$ \\
Overall agreement & 0.82 & 0.36 \\
PPV & 0.78 & 1.00 \\
NPV & 0.83 & 0.15 \\
AUC & $0.73(0.60-0.86)$ & $0.64(0.52-0.76)$ \\
\hline
\end{tabular}

Values with 95\% confidence intervals are shown. LAP was estimated by ECHO. LAP, left atrial pressure; BCG-B3, early diastolic ventricular vibration waves recorded by ballistocardiography; PPV, positive predictive value; NPV, negative predictive value; AUC, area under the ROC curve; ECHO, echocardiography.

value of 55.13 , the positive consistency value between the BCG-B3 index and the E/e' ratio in identifying LAP was 0.93 , the negative consistency value was 0.53 , the overall consistency value was 0.82 , the positive predictive value (PPV) was 0.78 , the negative predictive value (NPV) was 0.83 , and the AUC was 0.73 [95\% confidence interval (CI): $0.60-0.86 ; \mathrm{P}=0.0005]$. When the BCG-B3 index identified mildly and significantly elevated LAP, under a best cutoff value of 48.96 , the positive consistency value between the BCG-B3 index and the E/e' ratio in identifying LAP was 0.29 , the negative consistency value was 1.0 , the overall consistency value was 0.36 , the PPV was 1.0 , the NPV was 0.15 , and the $\mathrm{AUC}$ was 0.64 (95\% CI: $0.52-0.76$; $\mathrm{P}=0.03$ ). In summary, the diagnostic efficacy of the BCG-B3 index in identifying significantly elevated LAP was higher than that of the BCG-B3 index in identifying mildly elevated LAP.

\section{Discussion}

In this study, for the first time, the BCG-B3 index extracted by a BCG device using a fiber-optic sensor was compared with ECHO parameters, which are currently frequently used in clinical practice, and the BCG-B3 index showed a strong ability to identify high LAP. The current identification results of this study (only the BCG-B3 index was used) showed that the BCG-B3 index performed well, and the correlation coefficient between the BCG-B3 index and the E/e' ratio was 0.51 . Compared with the $\mathrm{E} / \mathrm{e}$ ' ratio in identifying LAP (17), the BCG-B3 index had a good identification ability for significantly elevated LAP, with an AUC of 0.73. Under the optimal cutoff value of 55.13, the positive consistency value between the BCG-B3 index and the E/e' ratio in identifying LAP was 0.93 , the negative consistency value was 0.53 , and the overall consistency value was 0.82 .

To better explore the diagnostic efficacy of the BCG-B3 index in identifying high LAP, an individual sign or symptom commonly used in the clinical assessment of volume overload and an individual ECHO parameter were used for comparison. Studies have shown (24) that in the clinical assessment of elevated filling pressure, an invasive right atrial pressure (IRAP) $>7 \mathrm{mmHg}$ is used as the standard. When jugular venous pulsation (JVP) $>8 \mathrm{~cm}$ is used as the cutoff value, the sensitivity is $48 \%$ and the specificity is $78 \%$; when the presence of jugular vein reflux is used as the threshold, the sensitivity is $50 \%$ and the specificity is $75 \%$; when liver enlargement is used, the sensitivity is $51 \%$ and the specificity was $62 \%$; and when edema in the legs is used, the sensitivity is $94 \%$ and the specificity was $10 \%$. The above methods have certain limitations. For example, observation of the jugular vein and physical examination of the liver are prone to the influence of obesity; for physical examination of liver enlargement and bilateral leg edema, false-positive identification may increase due to noncardiogenic reasons. $\mathrm{PCWP}>18 \mathrm{mmHg}$ is used as the gold standard. When dyspnea at rest is used as the threshold, the sensitivity is $50 \%$ and the specificity is $73 \%$; when dyspnea during exercise is used, the sensitivity is $66 \%$ and the specificity is $52 \%$; when orthopnea is used, the sensitivity is $66 \%$ and the specificity is $47 \%$; and when rales on auscultation is used, the sensitivity is $13 \%$ and the specificity is $90 \%$. These methods are susceptible 
to interference from other confounding factors, and dyspnea may be caused by noncardiac factors. In daily clinical practice, physicians strongly rely on ECHO to assess whether a patient has volume overload (25). In the assessment of filling pressure by ECHO, IRAP $>7 \mathrm{mmHg}$ is used as the gold standard. When inferior vena cava (IVC) collapse rate $\geq 50 \%$ is used as the cutoff value, the sensitivity is $12 \%$ and the specificity is $27 \%$; and when an IVC diameter $<12 \mathrm{~mm}$ is used, the sensitivity is $67 \%$ and the specificity is $91 \%$. This type of method is not suitable for patients with positive-pressure ventilation. PCWP $>18 \mathrm{mmHg}$ is used as the standard. When $\mathrm{E}>50(\mathrm{~cm} / \mathrm{s})$ is used as the cutoff value, the sensitivity is $92 \%$ and the specificity is $28 \%$; when a lateral E/e' ratio $>12$ is used, the sensitivity is $66 \%$ and the specificity is $55 \%$; when an E-wave deceleration time $\geq 130 \mathrm{~ms}$ is used, the sensitivity is $81 \%$ and the specificity is $80 \%$; when a ratio of peak pulmonary venous flow velocity during ventricular systole to that during ventricular diastole $(\mathrm{S} / \mathrm{D})<1$ is used, the sensitivity is $83 \%$ and the specificity is $72 \%$; and when the presence of diffuse $\mathrm{B}$-lines on lung ultrasound is used as the threshold, the sensitivity is $85.7 \%$ and the specificity is $40 \%$. In summary, based on the results of this study, the identification ability of the BCG-B3 index is superior to that of clinically used individual symptoms or signs. Because a non-gold standard was used as the reference in this study, the identification performance was reduced to some extent, and an accurate comparison of the BCG-B3 index with other individual ECHO parameters in identifying volume overload could not be performed.

According to the BCG characteristic curve, BCG-B3 occurs after S2 and before the ECG P wave, thus physiologically belonging to early diastole. The maximum amplitude recorded in this segment is defined as the BCG-B3 index. The BCG-B3 index substantially overlaps with $\mathrm{S} 3$ in the time phase. Regarding whether the BCG-B3 index recorded on and extracted from BCG traces belongs to $\mathrm{S} 3$, this study elaborated only the possibility of this parameter, and the specific mechanism requires further elucidation in subsequent animal experiments. S3 occurs in early diastole. At the end of rapid ventricular filling, mechanical vibration (including the ventricular wall, blood flow in the ventricular cavity, and adjacent structures) is generated due to rapid blood flow deceleration (26). When HF worsens, due to increased ventricular filling pressure and decreased myocardial compliance, $\mathrm{S} 3$ can be heard clearly $(26,27)$. S3 has moderate sensitivity and high specificity in identifying elevated LVFP $(28,29)$. S3 is considered one of the earliest symptoms of $\operatorname{HF}(26,27)$. Patients with $\mathrm{S} 3$ on auscultation during hospitalization have high all-cause and cardiogenic mortality (30). Clinical studies have shown that when S3 heard through a stethoscope by human ears is used to identify patients with elevated filling pressure, the sensitivity is 0.73 and the specificity is 0.42 . The positive consistency value between the $\mathrm{BCG}-\mathrm{B} 3$ index and the E/e' ratio was 0.93 , the negative consistency value was 0.53 , and the overall consistency value was 0.82 . Although the standard used was ECHO (which is widely used in clinical practice) rather than the gold standard, the current results showed that the BCG-B3 index performed well in identifying high LAP, suggesting the potential value of noninvasive wearable devices.

First, the good performance may benefit from the recorded low-frequency part of S3. S3 includes the audio part and infrasound part (low-frequency part) (31). Cardiac auscultation has been a useful clinical examination method for a long time. Traditionally, the audio frequency of S3 and other pathological heart sounds are usually determined by clinicians using a stethoscope. However, with the development of medicine, auscultation seems to be increasingly underused (32). The main reasons are as follows:

* Clinical experience: heart sound signals are complex and require well-trained professional medical personnel for accurate medical interpretations.

* Interobserver variation $(33,34)$ : the interpretation of heart sounds involves significant interobserver variation.

* Human hearing limitations (35): human can hear sound only between $20 \mathrm{~Hz}$ and $20 \mathrm{kHz}$. The frequency of diastolic vibration signals often exceeds this range; therefore, the human ear cannot hear them.

Although human ears cannot hear these vibrations due to physical limitations, these vibrations can be captured by sensors. Recent studies (31) have shown that the sensors in implanted cardiac devices can be used to monitor S3 amplitudes continuously (both audio and infrasound components) and provide objective quantification of $\mathrm{S} 3$, which can help remedy the aforementioned deficiencies. Thakur et al. reported that in animal models (16), the S3 intensity read by an implanted device was correlated with changes in LAP, and the correlation coefficient was 0.62 . The results of another study also showed that the S3 amplitude read by PCG had high specificity in identifying elevated LVEDP (28). In this study, the 
correlation coefficient between the BCG-B3 index and the $\mathrm{E} / \mathrm{e}$ ' ratio was 0.51 , which is a higher than those in animal experiments, and no invasive procedures are required.

Second, the good performance of this noninvasive device was due to the use of fiber-optic sensors. BCG uses highsensitivity fiber-optic sensor technology, which prevents interference from light on surrounding electromagnetic waves. Even extremely weak signals can be converted into effective electrical signals by increasing the light intensity (36). Therefore, compared with an ordinary sensor, fiber-optic sensors have higher sensitivity. In addition, most current devices for filling pressure monitoring must be implanted. For devices from traditional large medical device companies, such as Medtronic's cardiac resynchronization therapy (CRT) device Optivol (37) and Abbott's CRT device CorVue (38), transthoracic impedance (37,39-41) is often measured to understand pulmonary congestion, and then the occurrence of HF decompensation events can be predicted before symptom onset. However, these devices require patients to meet the indications for CRT and must be implanted. These devices are very expensive (the price can be hundreds of thousands of dollars), and the suitable patient population is small. The implantable cardioverter defibrillator/CRT with a defibrillator (ICD/CRT-D) belonging to the RESONATE family of Boston Scientific received US approval in 2017 and includes the HeartLogic function to warn of the onset of $\mathrm{HF}$ decompensation events, which is calculated using a series of composite parameters. Among the parameters, the two most important are S3 and the respiration rate. A follow-up of $900 \mathrm{HF}$ patients with the Boston Scientific ICD/CRT-D was conducted for 1 year. The HeartLogic function could provide early warnings of HF decompensation events by an average of 34 days in advance (42). However, the complications of ICD/CRT-D implantation include lead displacement, bleeding or infection of pacemaker sac, infective endocarditis, and inappropriate electric shock. For other minimally invasive implantable devices, such as CardioMEMS, 155 complications occurred from May 28, 2014, to May 28, 2017, including pulmonary embolism, device detachment, a need for reimplantation, pulmonary artery injury, and even death (43).

Finally, because of the enormous impact of HF on medical and health expenditures and quality of life, although the correlation coefficient between the BCG-B3 index and the E/e' ratio was 0.51 , the positive consistency value between the BCG-B3 index and the E/e' ratio was 0.93 , the negative consistency value was 0.53 , and the overall consistency value was 0.82 , this wearable device still has certain application prospects, which may generate significant benefits for the overall disease burden. In this study, the fiber-optic sensor-based BCG device measured and extracted the BCG-B3 index, which enables accurate and independent LAP determination in HF patients. This novel fiber-optic sensor-based device offers an opportunity for remote monitoring of HF patients using a continuous, objective, and reliable alternative method rather than hemodynamic examination.

\section{Study limitations}

At present, it's a novel method that we record BCG-B3 index by BCG technology with optical fiber sensor. Therefore, the BCG-B3 index has not been widely used in clinical practice. The main reasons are as follows. First, this study used the E/e' ratio (an ECHO parameter frequently used in clinical practice) as the reference rather than the gold standard, thus reducing the clinical value of the study results to some extent. Second, considering the complex pathophysiology of $\mathrm{HF}$, the manifestations of any special signs or symptoms are very different. An individual parameter may not be sufficient to assess the clinical status of a patient. In future studies, using the fiber-optic sensorbased BCG device, the BCG-B3 index will be combined with other physiological parameters, such as heart rate and respiratory rate, to establish a set of algorithms for comprehensive identification of elevated LAP, and the diagnostic value of an integrated algorithm for identifying elevated LAP in HF patients will be investigated.

\section{Conclusions}

The BCG-B3 index recorded by noninvasive BCG technology with a built-in fiber-optic sensor has important diagnostic value for identifying elevated LAP, and the accuracy is relatively high. BCG examination is not limited by venue and the doctor's experience. BCG can provide timely remote assessments for $\mathrm{HF}$ patients.

\section{Acknowledgments}

We thank our consultant Professor Carolyn Lam, Senior Consultant from the Department of Cardiology at the National Heart Centre Singapore (NHCS) who provided valuable insight and expertise on cardiac physiology and $\mathrm{HF}$, which helped us realize that comparing BCG signals 
with echocardiography parameters may be a good strategy to understand BCG and ultimately led to the beginning of this research. We would also like to extend our gratitude to Professor Jie Cheng, Director of Electrophysiology Basic Research Teaching Staff from the Texas Heart Institute. With his kind help, the engineering team from DARMA Technology Co., Ltd. and the clinical team from the First Affiliated Hospital of Shantou University Medical Colleges has the opportunity to work together in this research.

Funding: This study was funded by the 2019 Shantou Medical Science Talent Cultivation and Clinical Technology Promotion Project (190917105269872).

\section{Footnote}

Reporting Checklist: The authors have completed the STARD reporting checklist. Available at https://dx.doi. org/10.21037/apm-21-1840

Data Sharing Statement: Available at https://dx.doi. org/10.21037/apm-21-1840

Conflicts of Interest: All authors have completed the ICMJE uniform disclosure form (available at https://dx.doi. org/10.21037/apm-21-1840). All authors report this study was funded by the 2019 Shantou Medical Science Talent Cultivation and Clinical Technology Promotion Project (190917105269872). The authors have no other conflicts of interest to declare.

Ethical Statement: The authors are accountable for all aspects of the work in ensuring that questions related to the accuracy or integrity of any part of the work are appropriately investigated and resolved. All procedures performed in this study involving human participants were in accordance with the Declaration of Helsinki (as revised in 2013). This research project was approved by the Ethics Review Committee of the First Affiliated Hospital of Shantou University Medical College (No. 2019071). All patients provided written informed consent before participating in this clinical single-center diagnostic trial.

Open Access Statement: This is an Open Access article distributed in accordance with the Creative Commons Attribution-NonCommercial-NoDerivs 4.0 International License (CC BY-NC-ND 4.0), which permits the noncommercial replication and distribution of the article with the strict proviso that no changes or edits are made and the original work is properly cited (including links to both the formal publication through the relevant DOI and the license). See: https://creativecommons.org/licenses/by-nc-nd/4.0/.

\section{References}

1. Ponikowski P, Anker SD, AlHabib KF, et al. Heart failure: preventing disease and death worldwide. ESC Heart Fail 2014;1:4-25.

2. Hao G, Wang $X$, Chen $Z$, et al. Prevalence of heart failure and left ventricular dysfunction in China: the China Hypertension Survey, 2012-2015. Eur J Heart Fail 2019;21:1329-37.

3. Heidenreich PA, Albert NM, Allen LA, et al. Forecasting the impact of heart failure in the United States: a policy statement from the American Heart Association. Circ Heart Fail 2013;6:606-19.

4. Ponikowski P, Voors AA, Anker SD, et al. 2016 ESC Guidelines for the diagnosis and treatment of acute and chronic heart failure: The Task Force for the diagnosis and treatment of acute and chronic heart failure of the European Society of Cardiology (ESC) Developed with the special contribution of the Heart Failure Association (HFA) of the ESC. Eur Heart J 2016;37:2129-200.

5. Chaudhry SI, Wang Y, Concato J, et al. Patterns of weight change preceding hospitalization for heart failure. Circulation 2007;116:1549-54.

6. Ong MK, Romano PS, Edgington S, et al. Effectiveness of remote patient monitoring after discharge of hospitalized patients with heart failure: the Better Effectiveness After Transition -- Heart Failure (BEAT-HF) randomized clinical trial. JAMA Intern Med 2016;176:310-8.

7. Felker GM, Ahmad T, Anstrom KJ, et al. Rationale and design of the GUIDE-IT study: Guiding Evidence Based Therapy Using Biomarker Intensified Treatment in Heart Failure. JACC Heart Fail 2014;2:457-65.

8. Packer M, Abraham WT, Mehra MR, et al. Utility of impedance cardiography for the identification of shortterm risk of clinical decompensation in stable patients with chronic heart failure. J Am Coll Cardiol 2006;47:2245-52.

9. Abraham WT, Compton S, Haas G, et al. Intrathoracic impedance vs daily weight monitoring for predicting worsening heart failure events: results of the Fluid Accumulation Status Trial (FAST). Congest Heart Fail 2011;17:51-5.

10. Abraham WT, Stevenson LW, Bourge RC, et al. Sustained efficacy of pulmonary artery pressure to guide adjustment of chronic heart failure therapy: complete follow-up 
results from the CHAMPION randomised trial. Lancet 2016;387:453-61.

11. Bourge RC, Abraham WT, Adamson PB, et al. Randomized controlled trial of an implantable continuous hemodynamic monitor in patients with advanced heart failure: the COMPASS-HF study. J Am Coll Cardiol 2008;51:1073-9.

12. Ritzema J, Melton IC, Richards AM, et al. Direct left atrial pressure monitoring in ambulatory heart failure patients: initial experience with a new permanent implantable device. Circulation 2007;116:2952-9.

13. Inan OT, Baran Pouyan M, Javaid AQ, et al. Novel wearable seismocardiography and machine learning algorithms can assess clinical status of heart failure patients. Circ Heart Fail 2018;11:e004313.

14. Brahmbhatt DH, Cowie MR. Remote management of heart failure: an overview of telemonitoring technologies. Card Fail Rev 2019;5:86-92.

15. Siejko KZ, Thakur PH, Maile K, et al. Feasibility of heart sounds measurements from an accelerometer within an ICD pulse generator. Pacing Clin Electrophysiol 2013;36:334-46.

16. Thakur PH, An Q, Swanson L, et al. Haemodynamic monitoring of cardiac status using heart sounds from an implanted cardiac device. ESC Heart Fail 2017;4:605-13.

17. Nagueh SF, Smiseth OA, Appleton CP, et al. Recommendations for the evaluation of left ventricular diastolic function by echocardiography: an update from the American Society of Echocardiography and the European Association of Cardiovascular Imaging. J Am Soc Echocardiogr 2016;29:277-314.

18. Tolia S, Khan Z, Gholkar G, et al. Validating left ventricular filling pressure measurements in patients with congestive heart failure: CardioMEMS ${ }^{\mathrm{TM}}$ pulmonary arterial diastolic pressure versus left atrial pressure measurement by transthoracic echocardiography. Cardiol Res Pract 2018;2018:8568356.

19. Inan OT, Migeotte PF, Park KS, et al. Ballistocardiography and seismocardiography: a review of recent advances. IEEE J Biomed Health Inform 2015;19:1414-27.

20. BOZHENKO BS. Seismocardiography--a new method in the study of functional conditions of the heart. Ter Arkh 1961;33:55-64.

21. Sattar Y, Chhabra L. Electrocardiogram. In: StatPearls. Treasure Island: StatPearls Publishing, 2021.

22. Lee B. Review of the present status of optical fiber sensors. Optical Fiber Technology 2003;9:57-79.

23. Nagueh SF, Middleton KJ, Kopelen HA, et al. Doppler tissue imaging: a noninvasive technique for evaluation of left ventricular relaxation and estimation of filling pressures. J Am Coll Cardiol 1997;30:1527-33.

24. Mullens W, Damman K, Harjola VP, et al. The use of diuretics in heart failure with congestion - a position statement from the Heart Failure Association of the European Society of Cardiology. Eur J Heart Fail 2019;21:137-55.

25. Nagueh SF. Non-invasive assessment of left ventricular filling pressure. Eur J Heart Fail 2018;20:38-48.

26. Mehta NJ, Khan IA. Third heart sound: genesis and clinical importance. Int J Cardiol 2004;97:183-6.

27. Drazner MH, Rame JE, Stevenson LW, et al. Prognostic importance of elevated jugular venous pressure and a third heart sound in patients with heart failure. N Engl J Med 2001;345:574-81.

28. Marcus GM, Gerber IL, McKeown BH, et al. Association between phonocardiographic third and fourth heart sounds and objective measures of left ventricular function. JAMA 2005;293:2238-44.

29. Shah SJ, Michaels AD. Hemodynamic correlates of the third heart sound and systolic time intervals. Congest Heart Fail 2006;12 Suppl 1:8-13.

30. Minami Y, Kajimoto K, Sato N, et al. Third heart sound in hospitalised patients with acute heart failure: insights from the ATTEND study. Int J Clin Pract 2015;69:820-8.

31. Cao M, Gardner RS, Hariharan R, et al. Ambulatory monitoring of heart sounds via an implanted device is superior to auscultation for prediction of heart failure events. J Card Fail 2020;26:151-9.

32. Chizner MA. Cardiac auscultation: rediscovering the lost art. Curr Probl Cardiol 2008;33:326-408.

33. Ishmail AA, Wing S, Ferguson J, et al. Interobserver agreement by auscultation in the presence of a third heart sound in patients with congestive heart failure. Chest 1987;91:870-3.

34. Lok CE, Morgan CD, Ranganathan N. The accuracy and interobserver agreement in detecting the 'gallop sounds' by cardiac auscultation. Chest 1998;114:1283-8.

35. Manson AL, Nudelman SP, Hagley MT, et al. Relationship of the third heart sound to transmitral flow velocity deceleration. Circulation 1995;92:388-94.

36. Wu T, Liu G, Fu S, et al. Recent progress of fiber-optic sensors for the structural health monitoring of civil infrastructure. Sensors (Basel) 2020;20:4517.

37. Yu CM, Wang L, Chau E, et al. Intrathoracic impedance monitoring in patients with heart failure: correlation with fluid status and feasibility of early warning preceding 
hospitalization. Circulation 2005;112:841-8.

38. Heist EK, Herre JM, Binkley PF, et al. Analysis of different device-based intrathoracic impedance vectors for detection of heart failure events (from the Detect Fluid Early from Intrathoracic Impedance Monitoring study). Am J Cardiol 2014;114:1249-56.

39. Conraads VM, Tavazzi L, Santini M, et al. Sensitivity and positive predictive value of implantable intrathoracic impedance monitoring as a predictor of heart failure hospitalizations: the SENSE-HF trial. Eur Heart J 2011;32:2266-73.

40. van Veldhuisen DJ, Braunschweig F, Conraads V, et al. Intrathoracic impedance monitoring, audible patient alerts, and outcome in patients with heart failure. Circulation 2011;124:1719-26.

41. Böhm M, Drexler H, Oswald H, et al. Fluid status telemedicine alerts for heart failure: a randomized controlled trial. Eur Heart J 2016;37:3154-63.

42. Boehmer JP, Hariharan R, Devecchi FG, et al. A multisensor algorithm predicts heart failure events in patients with implanted devices: results from the MultiSENSE study. JACC Heart Fail 2017;5:216-25.

43. Vaduganathan M, DeFilippis EM, Fonarow GC, et al. Postmarketing adverse events related to the CardioMEMS HF system. JAMA Cardiol 2017;2:1277-9.

Cite this article as: Zhang L, Cai P, Deng Y, Lin J, Chu Z, Shi Q, Ye F, Hu J, Yang C, Li P, Zhuang S, Wang B. Ballistocardiography to identify high left atrial pressure in patients with heart failure. Ann Palliat Med 2021;10(7):8155-8168. doi: 10.21037/apm-21-1840 